\title{
Developing optofluidic technology through the fusion of microfluidics and optics
}

\author{
Demetri Psaltis ${ }^{1}$, Stephen R. Quake ${ }^{2} \&$ Changhuei Yang ${ }^{1}$
}

\begin{abstract}
We describe devices in which optics and fluidics are used synergistically to synthesize novel functionalities. Fluidic replacement or modification leads to reconfigurable optical systems, whereas the implementation of optics through the microfluidic toolkit gives highly compact and integrated devices. We categorize optofluidics according to three broad categories of interactions: fluid-solid interfaces, purely fluidic interfaces and colloidal suspensions. We describe examples of optofluidic devices in each category.
\end{abstract}

Optofluidics refers to a class of optical systems that are synthesized with fluids. Fluids have unique properties that cannot be found in solid equivalents, and these properties can be used to design novel devices. Examples of such properties include: the ability to change the optical property of the fluid medium within a device by simply replacing one fluid with another; the optically smooth interface between two immiscible fluids; and the ability of flowing streams of miscible fluids to create gradients in optical properties by diffusion. Most optical systems are currently made with solid materials such as glasses, metals and semiconductors, but there are cases in which it has been advantageous to use fluids. The oil-immersion microscope ${ }^{1}$, liquid mirrors for telescopes ${ }^{2}$, liquid-crystal displays ${ }^{3}$ and electrowetting lenses ${ }^{4}$ are good examples. Here we describe the various methods used to implement optofluidic devices with recently developed microfluidic technologies that allow accurate control of liquids on small spatial scales. Integration and reconfigurability are two major advantages associated with optofluidics. Whereas microfluidics has made it possible to integrate multiple fluidic tasks on a chip, most optical components, such as the light source, sensors, lenses and waveguides, remained off the chip. Optofluidic integration combines optics and microfluidics on the same chip by building the optics out of the same fluidic toolkit. The second advantage of optofluidics lies in the ease with which one can change the optical properties of the devices by manipulating fluids.

Microfluidics is a burgeoning field with important applications in areas such as biotechnology, chemical synthesis and analytical chemistry. Many of these applications of microfluidics are discussed elsewhere in this issue, and for the purposes of this Review we emphasize only a few salient points. First, there is now an extensive body of literature on how the physical properties of fluids that are available only on small spatial scales can be exploited for device functionality ${ }^{5-7}$. Many of these effects can also be used to control optical properties. Second, technological advances in device fabrication have made it possible to build miniaturized devices with complex networks of channels, valves, pumps and other methods of fluidic encapsulation and manipulation ${ }^{8}$. This creates a powerful set of tools for fluidic control, and because the feature sizes in these systems are shrinking over time, they will inevitably approach the wavelength of visible light. When this convergence happens, optofluidic-device applications will increase dramatically, just as the ability to lithographically define features with dimensions just below the wavelength of light has created a revolution in photonics. Third, a large anticipated market for microfluidics lies in portable devices for environmental monitoring, medical diagnostics
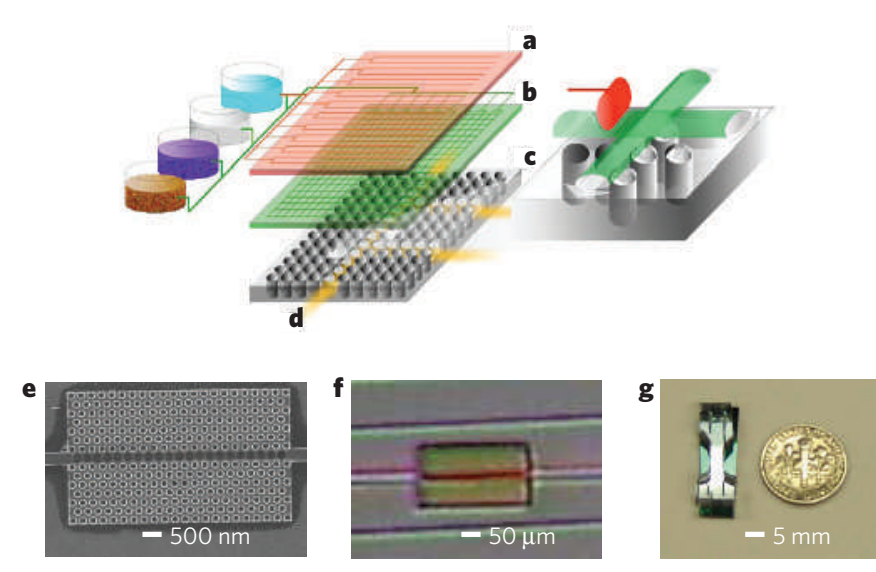

Figure 1 A generalized layer construction of an optofluidic device. An optofluidic device typically consists of three layers. a, The topmost layer consists of the microfluidic controls. Microfluidic valves and pumps may be incorporated in this layer. $\mathbf{b}$, The middle layer contains the microfluidic channels. c, The third layer is the optical structure and may contain photonic crystal structures, sensors, sources and waveguides. d, Light can be guided within the third layer. e-g, The range of scales ${ }^{23}$ involved in the fabricated device will typically span from subwavelength ( $\sim$ tens to hundreds of nanometres) for the optical structures to tens and hundreds of millimetres for the microfluidic structures.

and chemical-weapon detection. Optofluidics is helping to realize these aspirations by combining optical elements into microfluidic devices in ways that increase portability and sensitivity.

Although any microfluidic fabrication method can, in principle, be adapted to synthesize optofluidic devices, most of the implementations thus far have been with soft lithography ${ }^{9-11}$ (Fig. 1). Soft lithography allows rapid fabrication of complex microfluidic structures ${ }^{12}$ in flexible polymer substrates at a fraction of the cost of traditional glass or semiconductor manufacturing. Although the most popular polymer is poly(dimethylsiloxane) (PDMS), many other materials are suitable, such as photocurable hydrogels ${ }^{13}$, thermoset plastics ${ }^{14}$ and elastomers ${ }^{15}$, and photocurable solvent-resistant elastomers such as perfluoropolyethers $(\mathrm{PFPE})^{16}$. The optical transparency and good optical quality of PDMS has been demonstrated in applications such as soft lithographic fabrication of blazed gratings ${ }^{17}$ and solid immersion lenses ${ }^{18}$, showing that these materials are suitable in the optofluidic context. 
a

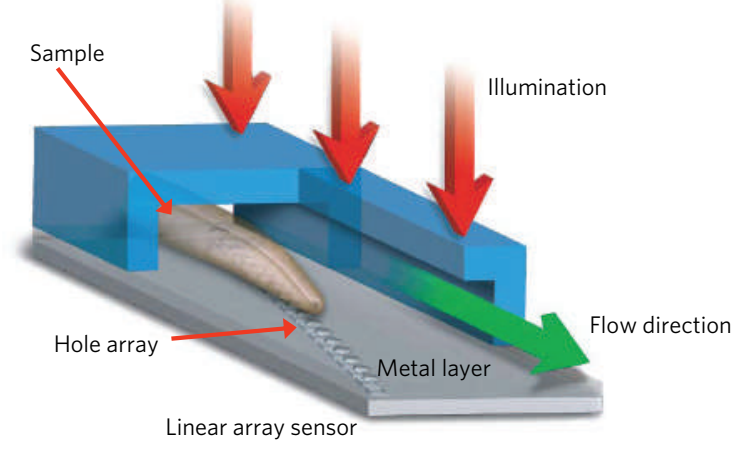

b

c

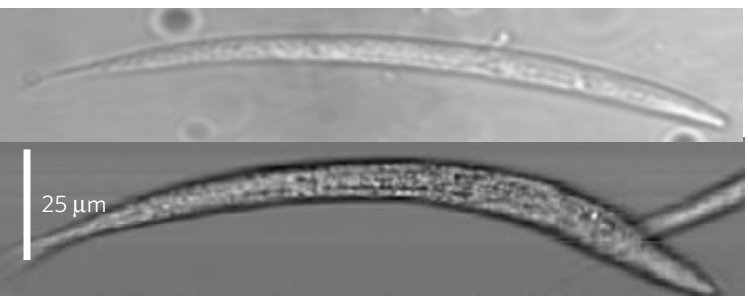

d

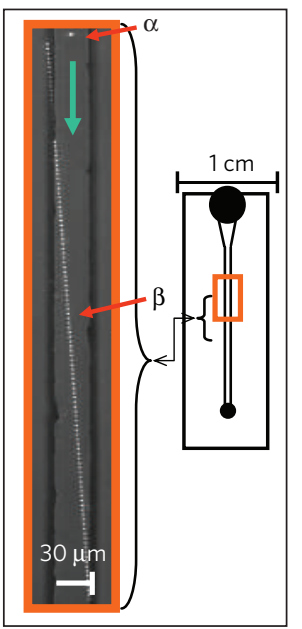

e

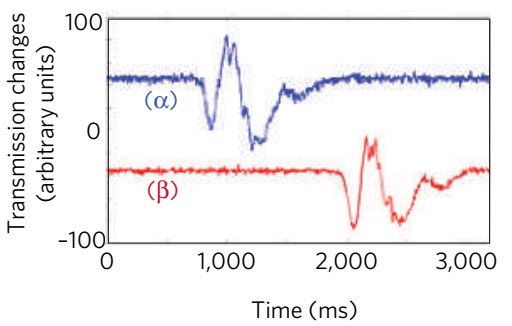

Figure 2 | The optofluidic microscope. a, Implementation scheme for an on-chip optofluidic microscope. The device is uniformly illuminated from the top. The target sample flows through the channel, and the transmission through each hole is acquired and recorded. The composition of the transmission traces creates a transmission image of the target sample. b, A conventional microscope image of Caenorhabditis elegans. c, An optofluidic microscope (700-nm resolution) image of C. elegans. d, By staggering the holes along the length of the channel, the separation between holes can be made equal to the pixel size of the underlying sensor array and enable the unique mapping of each hole to a pixel. The lateral displacement of the holes across the channel can be made arbitrarily small and it defines the resolution of the microscope. This approach enables the construction of microscopes with resolutions that are much finer than the pixel resolution of a conventional sensor grid. e, The transmission trace through two representative holes, $\alpha$ and $\beta$, on the microscope as the sample flows across them.

Optofluidic devices can be categorized into three major groups: structured solid-liquid hybrids in which the optical properties of both media are relevant; complete fluid-based systems in which only the optical properties of the fluids are relevant; and colloid-based systems in which manipulation of solid particles in liquid, or using the unique optical properties of colloidal solution, form the basis of the optofluidic devices. Recent progress in this last discipline has led to novel nanoparticles with interesting optical properties along with methods to control them in the solution phase.
Fluids in solids

The interface between materials with differing optical properties is commonly used for controlling the propagation of light. For example, the interface between air and a dispersive material such as glass in a prism separates the colours of an incident beam into different angles. Another important example can be found in fibre-optic waveguiding, in which total internal reflection at the interface between the cladding and a core with a higher index of refraction confines light to the core.

Interfaces between solid and liquid materials can also be used to synthesize optofluidic devices. This can be done by fabricating a solid structure with voids whose dimensions are larger than the wavelength of light. The reflection and transmission of light through the void is then modified by the insertion of liquids in the voids. Microfluidics and microfabrication provide the tools for making such devices, of which the Agilent switch ${ }^{19}$ is one of the earliest examples. In this case, inkjet technology was used to introduce or remove an index matching liquid from the back of a total internal reflection (TIR) mirror. The transmission of light could thus be switched on and off. More recently, Campbell et al. ${ }^{20}$ demonstrated an exchange-bypass switch using the same principle. Adaptive lenses can be formed in this way by varying the fluidic pressure in a soft PDMS encapsulation ${ }^{21,22}$. Finally, a periodic array of voids, in which each void can be selectively filled with absorbing dye, can be used to write an arbitrary two-dimensional pattern. This can therefore serve as a display or memory device. Such a device has been demonstrated by Quake's group using an extensive microfluidic circuit of channels and valves ${ }^{12}$.

Any adaptive device can be thought of as a sensor if the adaptation is due to something one wishes to characterize. In this regard, optofluidic adaptation mechanisms can often be used as sensors. For example, the compact tunable microfluidic interferometer described in ref. 23, in which a fluid-air interface is used to provide an optical path difference in a compact interferometer, can be adapted for biosensing or chemical analysis. The optofluidic microscope (OFM) is another good example ${ }^{24}$ (Fig. 2). Compiling the time-dependent changes of the optical transmission across a fluidic channel gives one the image of the sample flowing in the channel. The fact that the function of a microscope can be integrated into an optofluidic chip implies that multiple sensors can also be packed onto a compact chip and parallel processing of the sample be performed. Other examples of optofluidic sensors can be found in the compact resonant integrated microfluidic refractometer ${ }^{25}$ and fibre Bragg grating refractive-index sensors ${ }^{26}$.

A phenomenon related to total internal reflection, known as evanescent waves, also has applications in optofluidic devices. Evanescent waves are launched in the region of lower index when light undergoes total internal reflection at an interface. Even though evanescent modes cannot carry energy away from the interface, light propagation in the region of higher index can be strongly affected by the evanescent modes. This provides a non-intrusive mechanism for optofluidic control by introducing liquids with specific optical properties into the region surrounding the optical structure. For example, the phase velocity of a dielectric waveguide depends on the refractive index of the cladding. By selecting the index of the liquid that is inserted in the region surrounding the dielectric waveguide, we can adjust the phase delay through the waveguide. This has many applications in integrated optics, including optical switching and modulation. For instance, the adjustable phase delay has recently been used to tune a ring resonator that is constructed as a closed loop of a dielectric waveguide ${ }^{27}$ (Fig. 3). Besides phase-delay changes, the introduction of an absorbing liquid (a dye, for example) introduces attenuation of the light propagating in the waveguide. Optofluidic devices that rely on evanescent mode modification can also function as sensors. Surface-plasmon-based sensors ${ }^{28-30}$ are a well-known example of this. Other recent examples in this category include the optofluidic sensors based on the high-Q resonators developed by Vahala's group ${ }^{31}$ and the zero-mode waveguide sensors ${ }^{32}$.

In our discussion so far, we have assumed that the size of the voids is significantly larger than the optical wavelength. When the size of the voids is approximately equal to the wavelength, the effect of liquid insertion 

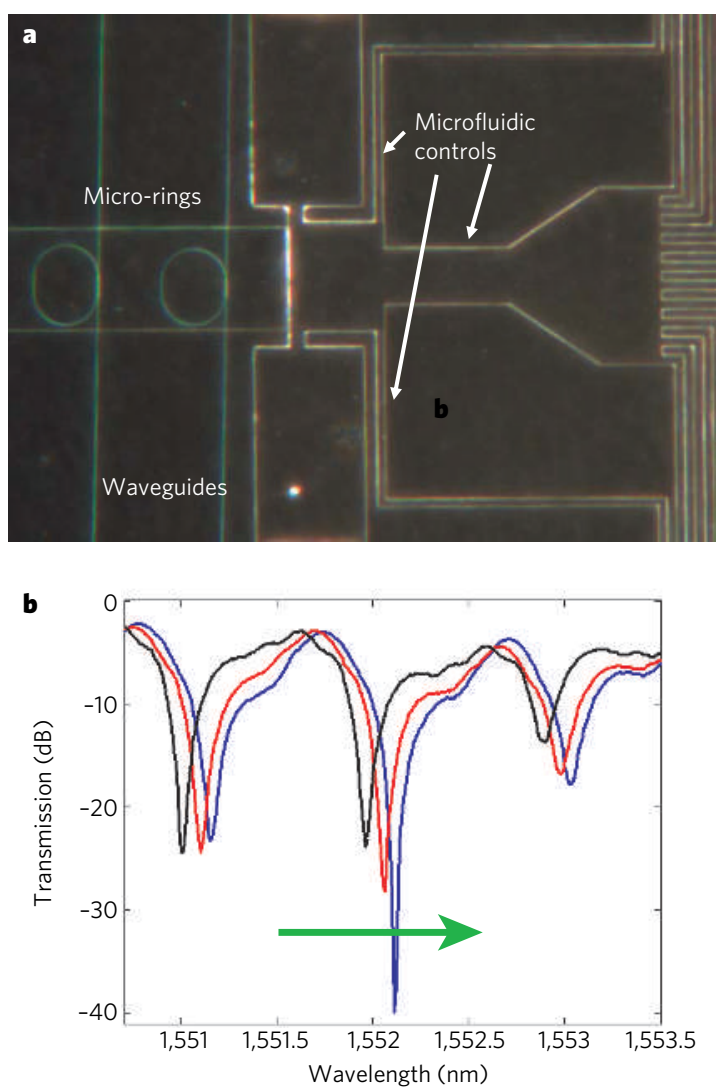

Figure 3 Optical filter based on an optofluidic micro-ring resonator. a, The optical structure consists of a bus waveguide coupled to a micro-ring waveguide resonator. Liquid in the microchannel constitutes the upper cladding of the waveguides. The refractive index of the liquid controls the resonance wavelength and the strength of the coupling. $\mathbf{b}$, At resonance, light travelling in the waveguide will be strongly attenuated. The graph shows that this resonance can be tuned by changing the refractive index of the liquid. The arrow indicates the increase in resonance wavelength as the refractive index increases. (Reprinted, with permission, from ref. 27.) can become more complex. For instance, a blazed grating immersed in liquid-crystal medium can be turned on and off by modifying the index of the liquid $^{33}$. As another example, consider a single-mode optical waveguide constructed by inserting liquid into a rectangular channel of the appropriate size. It would guide light in the liquid when the index of the liquid exceeds that of the surrounding medium. Such a liquid-core optofluidic waveguide is useful for measuring the properties of small volumes of liquid samples, because a long or highly structured waveguide can be easily realized $^{34}$. The idea can be further extended by introducing a periodic structure on the walls of the waveguide with period $\Lambda$. This periodic structure strongly reflects light with wavelengths equal to $2 n \Lambda / m$, where $m$ is a positive integer and $n$ is the index of refraction for the liquid. The frequency response of this filter can thus be tuned by modifying the index of the liquid. Such a structure has been used to show a distributed feedback dye laser ${ }^{35}$ (Fig. 4) - one of several optofluidic dye lasers that have been demonstrated recently ${ }^{36-38}$. As a class of optofluidic devices, these lasers are useful on their own and constitute an important set of enabling optofluidic platform tools. Among other advantages, these lasers are highly compact, widely tunable and robust. Their pump sources can be either an external light illumination or an on-chip laser diode illumination.

Photonic band gap (PBG) structures ${ }^{39}$ are particularly interesting for optofluidics because they naturally have voids into which fluids can be injected. A completely uniform PBG structure is of little practical interest. It is the introduction of 'defects' that gives PBGs interesting functionalities, such as waveguiding capabilities or resonances. A defect consists of one or several voids in the periodic structure; typically, the size of the void varies and is defined lithographically when the device is fabricated. In the context of optofluidics, defects can be easily created or reconfigured by filling specific voids with liquid ${ }^{40}$. PBG structures can thus be reconfigured optofluidically by interfacing the photonic and microfluidic circuits. An integrated fluidic/photonic device that fluidically switches a PBG waveguide ${ }^{41}$ has recently been demonstrated (Fig. 1). In addition, a PBG cavity can be formed by a single defect. The resonant frequency of the PBG structure can be tuned by the liquid inserted at the defect site $^{42}$. Such cavities have been used to build PBG lasers ${ }^{43,44}$, sensors ${ }^{45}$ and slow-light structures ${ }^{46}$. PBG fibres ${ }^{47}$ are of great interest for optofluidics as well, because the cladding or the core can contain hollow regions into which fluids can be inserted, thereby modifying a fibre's transmission properties $^{34,48}$.

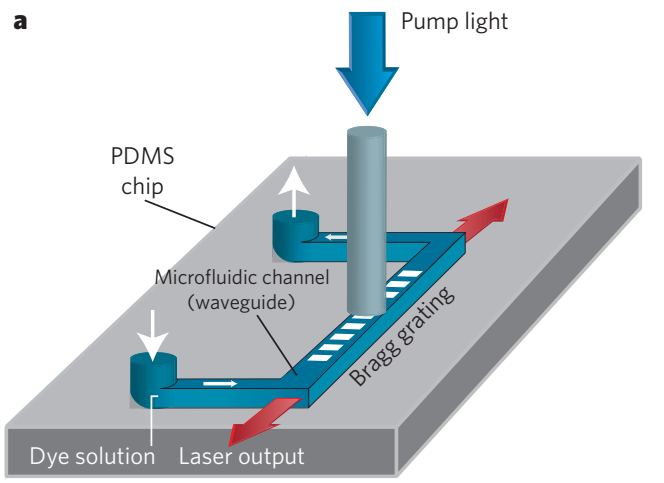

b

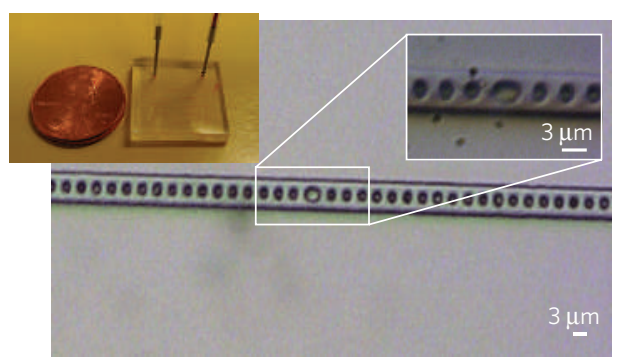

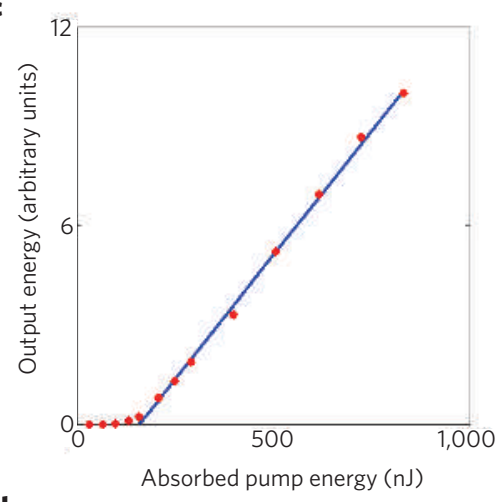

d

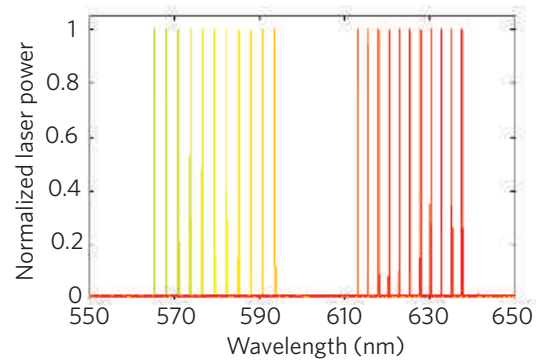

Figure 4 | An optofluidic distributed feedback (DFB) dye laser. a, A schematic of the laser. A microfluidic channel with a distributed feedback Bragg grating structure is fabricated in PDMS. A dye solution, which acts as both the core of the optical waveguide and the gain medium, can be introduced into the structure through the channel. An excitation pump light field is incident on the laser structure. b, An optical micrograph of the DFB structure. The laser's wavelength may be tuned by changing the dye choice or by stretching/compressing the flexible PDMS to change the periodicity of the DFB structure. Insert shows a device next to a penny to indicate its small size. c, Experimental data demonstrating the threshold characteristic of the laser. d, Demonstration of $\sim 60 \mathrm{~nm}$ tuning range for the laser that can be achieved by mechanically deforming the structure and changing the dye choice (yellow, Rhodamine 6G dye; red, Rhodamine 101 dye). 
Looking forward into the near future we can expect to see optofluidic systems that are based on porous solid systems in which sub-wavelength voids or pores are randomly distributed. In such systems, the voids do not scatter light but collectively modify the effective index of the homogeneous material. Insertion of a liquid into the voids can tune the refractive index, absorption and birefringence ${ }^{49}$ of such systems.
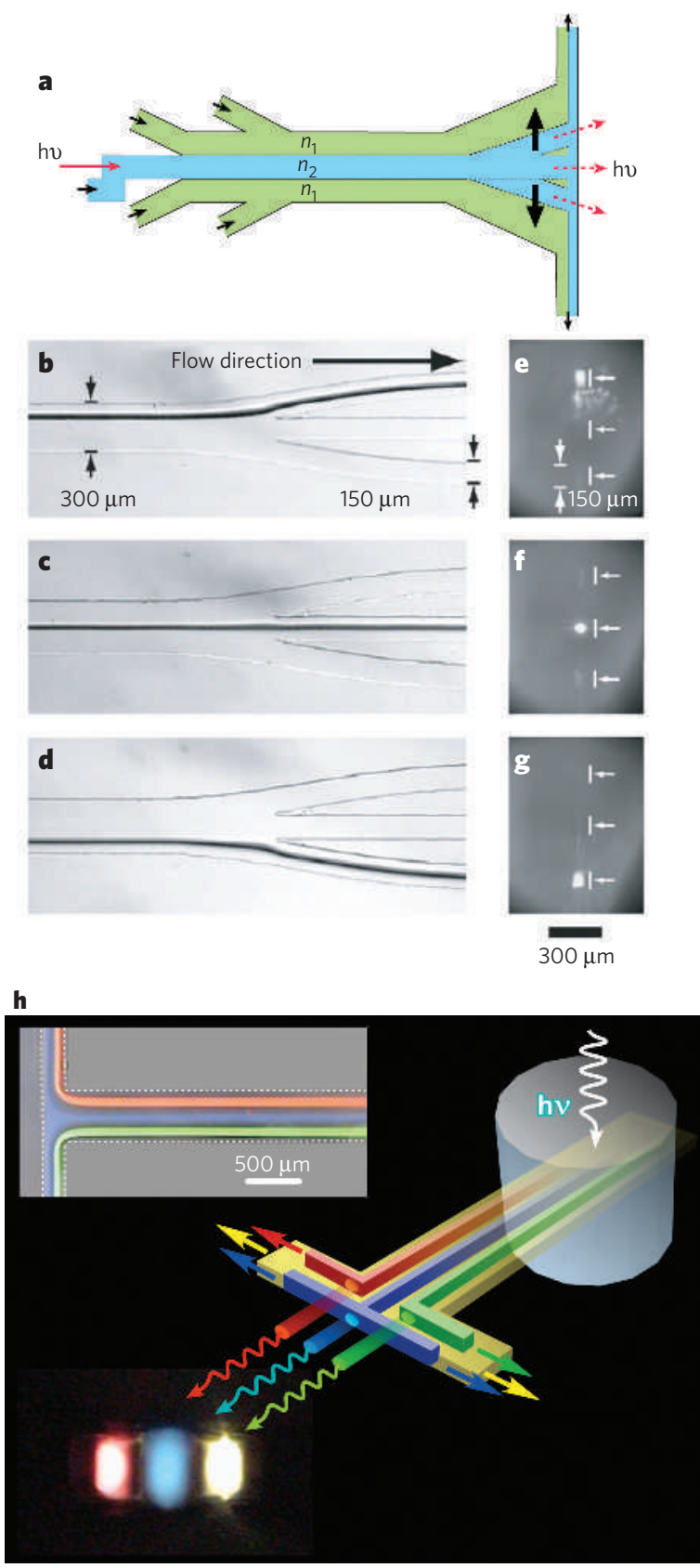

Figure 5 | The liquid-core/liquid-cladding $\left(L^{2}\right)$ waveguide. a, Schematic of an $L^{2}$ waveguide. The green and blue regions represent two different liquids. By choosing the inner-core liquid to be of higher refractive index $\left(n_{2}\right)$ than the refractive index $\left(n_{1}\right)$ of the surrounding media, light can be guided within the inner core. The red arrows indicate the direction of light input and propagation. $\mathrm{h} v$ is the energy quantum of the light. b-d, The direction of light propagation can be easily altered by altering the differential flow rates for the liquids to steer the inner-core liquid. e-g, Experimental verification of light output switching via fluid-core steering. $\mathbf{h}$, By channelling different fluorescent dyes in the waveguide, it is possible to create microfluidically controllable light sources. (Reprinted, with permission, from ref. 67.)

\section{Fluids in fluids}

Our discussion thus far has focused on the use of different combinations of solids and liquids to elicit changeable optical effects. The replacement of the solid substrate with a liquid in an optofluidic system can result in an even greater degree of flexibility. Whitesides' group's work on liquidcore/liquid-cladding $\left(L^{2}\right)$ optical waveguides is an excellent example of such a system ${ }^{50}$. An $L^{2}$ waveguide typically consists of a microfluidic channel in which an optically dense fluid flows within an enveloping sheath of fluid with a lower refractive index (Fig. 5). In addition to serving as waveguides, this method can also be used to create tunable light sources by the addition of dyes to the core fluid ${ }^{51}$.

Purely fluidic systems have several unique advantages that cannot be obtained from solid-fluid hybrids. First, the physical profile of the fluidic system that is optically relevant can be quite independent of the actual profile of the underlying microfluidic system. For example, in the case of the $L^{2}$ waveguide, light travelling through this dynamic waveguide can be directed and steered by simply changing the relative flow profile and flow rate of the fluid ${ }^{50}$. Second, and perhaps more importantly, it is possible to achieve finer features in the composite fluid media. For example, ref. 50 reported the establishment of a $<10 \mu \mathrm{m}$ jet stream that was formed in a large $(>100 \mu \mathrm{m})$ microfluidic channel. Finally, the interface between the two fluids can be made optically smooth by either choosing two immiscible fluids or by flowing the fluids next to each other at low Reynolds numbers. The roughness of the underlying microfluidic architecture has little impact on the fluid interfaces.

It has long been recognized that the optical smoothness of fluid interfaces can be a useful and cost-effective way to circumvent the challenges of creating surfaces of similar quality in solid systems. Perhaps even more interesting in the optical context is the fact that the meniscus between two immiscible fluids of equal density in a column is perfectly spherical - a curvature profile that is used in most commercially available lenses. An example of how this optically relevant interface has been made use of can be found in the variable-focus liquid lens that was developed by Philips Research Eindhoven ${ }^{4}$. The lens consists of two immiscible liquids of different refractive indices placed in a cylindrical housing. The lens is designed in such a way that the curvature of the meniscus and thus the effective focal length of the lens can be altered by electrowetting ${ }^{52}$. The same physical mechanism has also been used for displays by modifying the transmittance of a pixel through the movement of a dye solution ${ }^{53}$.

Miscible liquids and their interfaces can also be of significant use in the optofluidic context ${ }^{54}$. The diffusion across the interface of two liquids is another unique property that doesn't have a direct equivalent in solidbased devices. Specifically, the diffusion process can create a concentration and refractive-index gradient. The controllability and flexibility by which this gradient can be adjusted through flow parameters and fluid choices enable the creation of novel optical interconnects. For example, an optical splitter and wavelength filter based on the selective mixing of two fluid jets in a third fluidic medium has been demonstrated ${ }^{55}$. Unlike a conventional beamsplitter, the split ratio of the optofluidic beamsplitter can be dynamically tuned for any given wavelength by changing the absorbance properties of the input fluidic jets.

\section{Solids in fluids}

There is a third possibility that is optically interesting: the introduction of solid particles in a fluid. Such colloidal optofluidic systems can enable large changes in the optical properties of the resulting medium. Furthermore, small particles or colloids within the fluid can be manipulated with relative ease and precision, thereby creating gradients in the optical properties or very localized modifications. The ramifications of such manipulations in terms of synthesizing novel optical functionality are wide ranging and exploration of these has only begun recently.

Small particles are optically interesting in various ways. For example, dielectric particles of size comparable to the wavelength can exhibit significant spectral and angular scattering variations ${ }^{56}$. Particles that are significantly smaller than the wavelength can be used to change the refractive index or absorption coefficient of the medium as a whole $e^{56}$. 

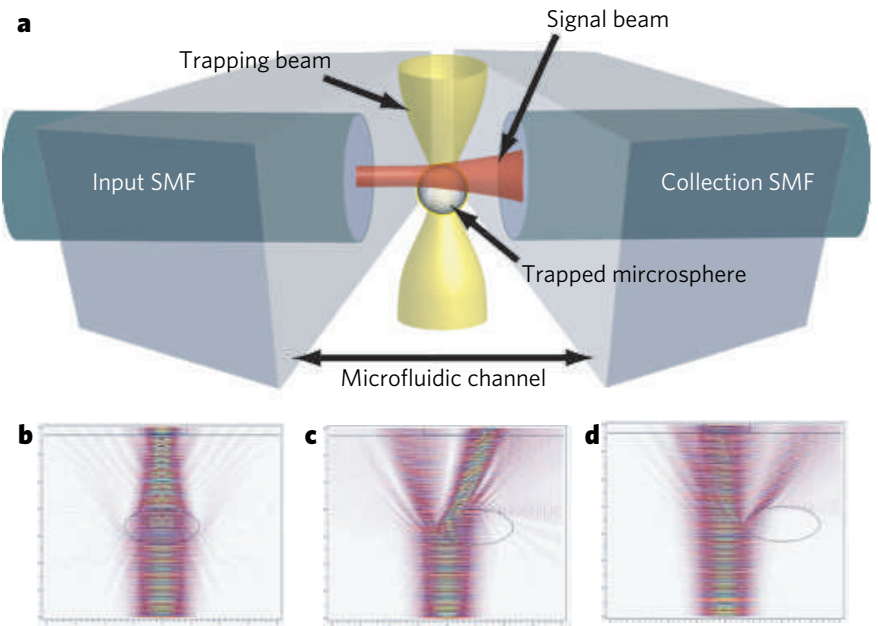

Figure 6 | The all-optical switch based on optofluidic beam manipulation. a, Schematic of the switch. The trapping beam steers a microsphere between two single-mode fibres (SMFs). The extent of coupling between the two fibres depends on the position of the microsphere. On the basis of its position, the microsphere can either focus the light from one fibre into the next, or deflect it away. b-d, Simulation illustrating that the beam deflection depends on the microsphere displacement.

Particles that are significantly larger than the wavelength can be treated as a spherical focusing lens in the medium ${ }^{57,58}$. Quantum dots can be designed to fluoresce or scatter with dramatically improved efficiency ${ }^{59}$. It is also interesting to note that quantum dots can be designed to fluoresce over a very narrow ( tens of nanometres) and pre-determined spectral bandwidth. Recently developed crescent-shaped nanoparticles have enhanced Raman scattering cross-sections ${ }^{60}$ and other optically interesting properties ${ }^{61}$.

There are several methods for optically manipulating the particles. Optical tweezing, one of the more established and popular methods, has seen significant improvements over the past decade. At present, up to 400 particles can be simultaneously manipulated ${ }^{62,63}$. In addition to optical tweezing, particles can be transported in a fluid through electric fields if they are charged, or through electric field gradients if they have a different electric permittivity from the surrounding medium. Fluidic flows can also be used to transport and affect the particle concentration. Finally, if ferromagnetic layers are included in the construction of the particles, magnetic fields can control their orientation and trajectory. A particularly interesting method was recently developed in which both light and electric fields are combined to effect particle manipulation ${ }^{64}$. In this approach, an optical image is projected onto a specially prepared glass substrate. The light field activates the coated photoconductive layer and creates non-uniform electric fields. These fields can either attract or repel particles that are in the solution above the substrate. This approach of optofluidic particle manipulation is especially noteworthy in terms of the number of particles that can be manipulated simultaneously - 15,000 traps were created over an area of about $1 \mathrm{~mm}^{2}$ in the demonstration experiment.

The ability to optically control particles can be used to create a range of novel optofluidic devices. A clear and elegant example is the optofluidic beam manipulator demonstrated by Dumachuk et al. ${ }^{57}$. The general concept is as follows. An optically trapped microsphere that is placed in front of the exit port of a waveguide can function as an optically movable lens for beam manipulation. By steering the microsphere, the output beam can be deflected in a range of directions. Dumachuk et al. ${ }^{56}$ used the method to create an all-optical switch by steering the microsphere between two mutually facing waveguides that are separated by a short distance (Fig. 6). The transmission of light from one waveguide to the other is enhanced when the microsphere is well centred and functions as a focussing lens. Individual particles can also be used to store bits of information. In a recent demonstration, a nanoparticle memory was synthesized by electrically transporting quantum dots to selected sites in a substrate. The spectral composition of the quantum-dot cocktails represents the stored information (Fig. 7).

The simple presence of the particles in a solution modifies its optical properties. This effect was used to demonstrate a liquid-liquid waveguide in which the core is a colloidal suspension surrounded by a cladding of pure solvent. By changing the diameter and volume fraction of the particles, it was possible to alter the propagation properties of the waveguide. The presence of nanoparticles in a liquid also affects the nonlinear optical properties of the medium. For example, the plasmon resonance of metal nanoparticles enhances the field locally by two or three orders of magnitude. The increase in the field strength leads to an enhancement of the nonlinear response of the medium, a property that has been used for surface-enhanced Raman scattering. Thermal nonlinearities introduced by metal nanoparticles in a solvent were recently used to demonstrate holographic recording in a fluid ${ }^{65}$.

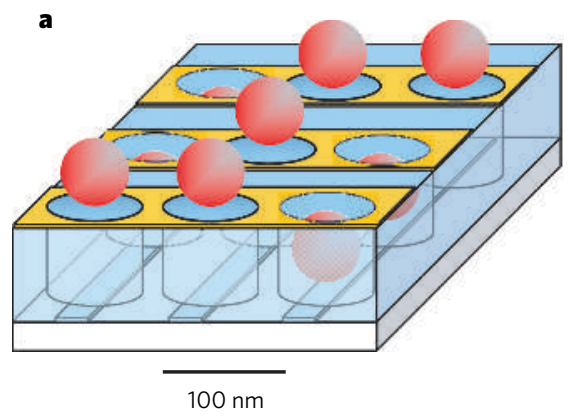

c

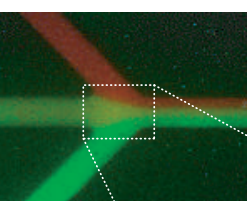

b

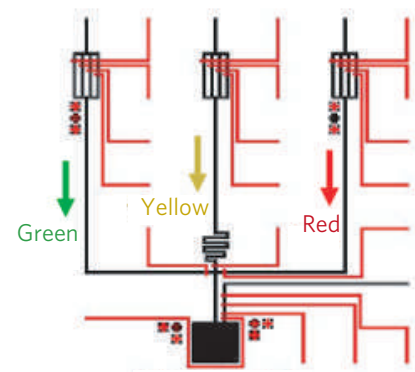

d

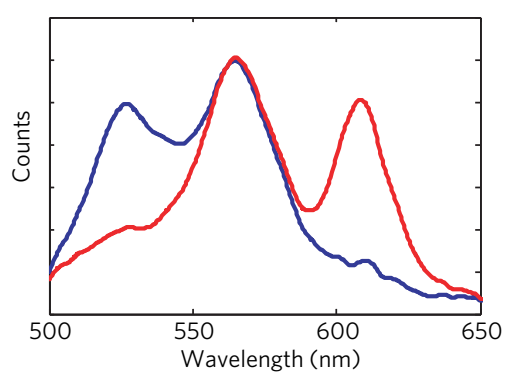

Figure $7 \mid$ An optofluidic memory system based on nanowells and quantum dots. a, An array of electrically addressable nanowells is used to trap quantum dots with resolution smaller than an optical wavelength. Data can be encoded by the presence or absence of photoluminescence in each region, or by spectral codes consisting of cocktails of quantum dots with distinct emission spectra in a single region. $b$, The microfluidic mixing circuit used to supply multiple quantum dots with different photoluminescence wavelengths to the nanowell array. c, A fluorescence image of the mixing of three species of quantum dots. Here the flow rate of each channel can be controlled to vary the relative concentrations of the types of dot, to generate distinct spectral codes. $d$, A spectrogram of two separate codes produced by the mixing circuit. Each peak in the spectrum represents a single base $M$ digit, where $M$ is the number of distinguishable intensity levels. A memory with $N$ species of quantum dots gives an information density of $N \log _{2}(M)$ bits per nanowell. (Reprinted, with permission, from ref. 68.) 
The ability to optically manipulate colloidal particles can be used as a mechanism for optically controlling the fluidic flow. An example of this is the recent work by Lee's group ${ }^{66}$. In this work, the enhanced optical absorption and subsequent heat generation of metallic nanoparticles is used. The method is simple and direct. Nanoparticles are added to the liquid of interest. A light beam is then introduced at the air-liquid interface. Due to the enhanced optical absorption and heat generation, the liquid evaporates and condenses beyond the edge. Subsequent condensation of the droplets wets the region adjacent to the air-liquid interface and causes a shift of the entire liquid body.

\section{Looking forward}

We conclude by noting that optofluidics is a field still in its infancy. It is full of promise, but few devices have been successfully commercialized as of yet. Many technological elements and even some fundamental concepts are just now being developed. As technological tools, optofluidic devices are good complements to micro-electromechanical systems (MEMS). Where MEMS physically actuate solid components to achieve reconfigurability, optofluidic devices reconfigure themselves through fluidic controls. Finally, as we have described in this Review, the synergy between optics and fluids is particularly rich. One currently understudied area that is especially noteworthy is the optical control of fluids. Such controls can dramatically simplify fluid manipulation by completely removing the need for on-chip plumbing.

1. Ronchi, V. Giovan Battista Amici's contribution to the advances of optical microscopy. Physis 11, 520-533 (1969).

2. Wood, R. W. Astrophys. J. The mercury paraboloid as a reflecting telescope. 29, 164-176 (1909).

3. Haas, W. E. Liquid-crystal display research — the first 15 years. Mol. Cryst. Liq. Cryst. 94, 1-31 (1983)

4. Kuiper, S. \& Hendriks, B. H. W. Variable-focus liquid lens for miniature cameras. Appl. Phys. Lett. 85, 1128-1130 (2004).

5. Squires, T. M. \& Quake, S. R. Microfluidics: fluid physics at the nanoliter scale. Rev. Mod. Phys. 77, 977-1026 (2005).

6. Stone, H. A., Stroock, A. D. \& Ajdari, A. Engineering flows in small devices: microfluidics toward a lab-on-a-chip. Annu. Rev. Fluid Mech. 36, 381-411 (2004).

7. Atencia, J. \& Beebe, D. J. Controlled microfluidic interfaces. Nature 437, 648-655 (2005)

8. Hong, J. W. \& Quake, S. R. Integrated nanoliter systems. Nature Biotechnol. 21, 1179-1183 (2003)

9. Duffy, D. C., McDonald, J. C., Schueller, O. J. A. \& Whitesides, G. M. Rapid prototyping of microfluidic systems in poly(dimethylsiloxane). Analyt. Chem. 70, 4974-4984 (1998).

10. Quake, S. R. \& Scherer, A. From micro- to nanofabrication with soft materials. Science 290, 1536-1540 (2000).

11. McDonald, J. C. et al. Fabrication of microfluidic systems in poly(dimethylsiloxane). Electrophoresis 21, 27-40 (2000).

12. Thorsen, T., Maerkl, S. J. \& Quake, S. R. Microfluidic large-scale integration. Science 298, 580-584 (2002).

13. Beebe, D. J. et al. Microfluidic tectonics: a comprehensive construction platform for microfluidic systems. Proc. Natl Acad. Sci. USA 97, 13488-13493 (2000)

14. Fiorini, G. S., Lorenz, R. M., Kuo, J. S. \& Chiu, D. T. Rapid prototyping of thermoset polyester microfluidic devices. Anal. Chem. 76, 4697-4704 (2004).

15. Sudarsan, A. P., Wang, J. \& Ugaz, V. M. Thermoplastic elastomer gels: an advanced substrate for microfluidic chemical analysis systems. Anal. Chem. 77, 5167-5173 (2005).

16. Rolland, J. P., Van Dam, R. M., Schorzman, D. A., Quake, S. R. \& DeSimone, J. M. Solvent resistant photocurable 'liquid teflon for microfluidic device fabrication'. J. Am. Chem. Soc. 126, 2322-2323 (2004).

17. Xia, Y. N. et al. Complex optical surfaces formed by replica molding against elastomeric masters. Science 273, 347-349 (1996).

18. Gambin, Y., Legrand, O.\& Quake, S. R. Microfabricated rubber microscope using soft solid immersion lenses. Appl. Phys. Lett. 88, 174102 (2006).

19. Introducing the Agilent Photomic Switching Platform [online] <http://physics.pdx. edu/ larosaa/Agilent_All_Optical_Network.htm>

20. Campbell, K. et al. A microfluidic $2 \times 2$ optical switch. Appl. Phys. Lett. 85, 6119-6121 (2004).

21. Pang, L., Levy, U., Campbell, K., Groisman, A. \& Fainman, Y. Set of two orthogonal adaptive cylindrical lenses in a monolith elastomer device. Opt. Express 13, 9003-9013 (2005).

22. Zhang, D. Y., Justis, N. \& Lo, Y. H. Fluidic adaptive lens of transformable lens type. Appl. Phys. Lett. 84, 4194-4196 (2004).

23. Grillet, C. et al. Compact tunable microfluidic interferometer. Opt. Express 12, 5440-5447 (2004).

24. Heng, X. et al. Optofluidic microscopy - a method for implementing a high resolution optical microscope on a chip. Lab Chip (submitted).

25. Domachuk, P., Littler, I. C. M., Cronin-Golomb, M. \& Eggleton, B. J. Compact resonant integrated microfluidic refractometer. Appl. Phys. Lett. 88, 093513 (2006).

26. Liang, W., Huang, Y. Y., Xu, Y., Lee, R. K. \& Yariv, A. Appl. Phys. Lett. 86, 151122 (2005).

27. Levy, U., Campbell, K., Groisman, A., Mookherjea, S. \& Fainman, Y. On-chip microfluidic tuning of an optical microring resonator Appl. Phys. Lett. 88, 111107-111109 (2006).

28. Homola, J., Yee, S. S. \& Gauglitz, G. Surface plasmon resonance sensors: review. Sens. Actuators B Chem. 54, 3-15 (1999).
29. Schuck, P. Use of surface plasmon resonance to probe the equilibrium and dynamic aspects of interactions between biological macromolecules. Annu. Rev. Biophys. Biomol. Struct. 26, 541-566 (1997).

30. Vo-Dinh, T. Surface-enhanced Raman spectroscopy using metallic nanostructures. Trends Analyt. Chem. 17, 557-582 (1998)

31. Armani, A. M., Armani, D. K., Min, B., Vahala, K. J. \& Spillane, S. M. Ultra-high-Q microcavity operation in $\mathrm{H}_{2} \mathrm{O}$ and $\mathrm{D}_{2} \mathrm{O}$. Appl. Phys. Lett. 87, 151118 (2005).

32. Levene, M. J. et al. Zero-mode waveguides for single-molecule analysis at high concentrations. Science 299, 682-686 (2003).

33. Wang, X., Wilson, D., Muller, R., Maker, P. \& Psaltis, D. Liquid-crystal blazed-grating beam deflector. Appl. Opt. 39, 6545-6555 (2000).

34. Mach, P. et al. Tunable microfluidic optical fiber. Appl. Phys. Lett. 80, 4294-4296 (2002).

35. Li, Z. Y., Zhang, Z. Y., Emery, T., Scherer, A. \& Psaltis, D. Single mode optofluidic distributed feedback dye laser. Opt. Express 14, 696-701 (2006)

36. Galas, J. C., Torres, J., Belotti, M., Kou, Q. \& Chen, Y. Microfluidic tunable dye laser with integrated mixer and ring resonator. Appl. Phys. Lett. 86, 264101 (2005)

37. Vezenov, D. V. et al. A low-threshold, high-efficiency microfluidic waveguide laser. J. Am. Chem. Soc. 127, 8952-8953 (2005).

38. Helbo, B., Kragh, S., Kjeldsen, B. G., Reimers, J. L. \& Kristensen, A. Investigation of the dye concentration influence on the lasing wavelength and threshold for a microfluidic dye laser Sens. Actuators A Phys. 111, 21-25 (2004).

39. Joannopoulos, J. D., Meade, R. D. \& Winn, J. N. Photonic Crystals (Princeton Univ. Press, Princeton, New Jersey, 1995).

40. Domachuk, P., Nguyen, H. C., Eggleton, B. J., Straub, M. \& Gu, M. Microfluidic tunable photonic band-gap device. Appl. Phys. Lett. 84, 1838-1840 (2004).

41. Erickson, D., Rockwood, T., Emery, T., Scherer, A. \& Psaltis, D. Nanofluidic tuning of photonic crystal circuits. Opt. Lett. 31, 59-61 (2006)

42. Maune, B. et al. Liquid-crystal electric tuning of a photonic crystal laser. Appl. Phys. Lett. $\mathbf{8 5}$ 360-362 (2004).

43. Painter, O. et al. Two-dimensional photonic band-gap defect mode laser. Science $\mathbf{2 8 4}$, 1819-1821 (1999)

44. Dowling, J. P., Scalora, M., Bloemer, M. J. \& Bowden, C. M. The photonic band edge laser: a new approach to gain enhancement. J. Appl. Phys. 75, 1896-1899 (1994).

45. Shinn, A. \& Robertson, W. M. Surface plasmon-like sensor based on surface electromagnetic waves in a photonic band-gap material. Sens. Actuators B Chem. 105, 360-364 (2005)

46. Mookherjea, S. \& Yariv, A. Coupled-resonator optical waveguides. IEEE J. Select. Topics Quantum Electron. 8, 448-456 (2002)

47. Knight, J. C., Broeng, J., Birks, T. A. \& Russel, P. S. J. Photonic band gap guidance in optical fibers. Science 282, 1476-1478 (1998).

48. Cattaneo, F. et al. Digitally tunable microfluidic optical fiber devices. J. Microelectromech. Syst. 12, 907-912 (2003).

49. Xu, F. et al. Fabrication, modeling, and characterization of form-birefringent nanostructures. Opt. Lett. 20, 2457-2459 (1995).

50. Wolfe, D. B. et al. Dynamic control of liquid-core/liquid-cladding optical waveguides. Proc Natl Acad. Sci. USA 101, 12434-12438 (2004).

51. Vezenov, D. V., Mayers, B. T., Wolfe, D. B.\& Whitesides, G. M. Integrated fluorescent light source for optofluidic applications. Appl. Phys. Lett. 86, 041104 (2005).

52. Mugele, F. \& Baret, J. C. Electrowetting: from basics to applications. J. Phys. Condens. Matter 17, R705-R774 (2005)

53. Hayes, R. A. \& Feenstra, B. J. Video-speed electronic paper based on electrowetting. Nature 425, 383-385 (2003)

54. Groisman, A., Enzelberger, M. \& Quake, S. R. Microfluidic memory and control devices. Science 300, 955-958 (2003).

55. Wolfe, D. B. et al. Diffusion-controlled optical elements for optofluidics. Appl. Phys. Lett. 87, 181105 (2005).

56. van der Hulst, H. C. Light Scattering by Small Particles (Dover Publications, New York, 1957).

57. Domachuk, P. et al. Application of optical trapping to beam manipulation in optofluidics. Opt. Express 13, 7265-7275 (2005).

58. Brody, J. P. \& Quake, S. R. A self-assembled microlensing rotational probe. Appl. Phys. Lett. 74, 144-146 (1999).

59. Trindade, T., O'Brien, P. \& Pickett, N. L. Chem. Mater. 13, 3843-3858 (2001).

60. Lu, Y., Liu, G. L., Kim, J., Mejia, Y. X. \& Lee, L. P. Nanophotonic crescent moon structures with sharp edge for ultrasensitive biomolecular detection by local electromagnetic field enhancement effect. Nano Lett. 5, 119-124 (2005).

61. Aizpurua, J. et al. Optical properties of gold nanorings. Phys. Rev. Lett. 90, 057401 (2003).

62. Neuman, K. C. \& Block, S. M. Optical trapping. Rev. Sci. Instrum. 75, 2787-2809 (2004).

63. Grier, D. G. A revolution in optical manipulation. Nature 424, 810-816 (2003).

64. Chiou, P. Y., Ohta, A. T. \& Wu, M. C. Massively parallel manipulation of single cells and microparticles using optical images. Nature 436, 370-372 (2005).

65. Adleman, J. R., Eggert, H. A., Buse, K. \& Psaltis, D. Holographic grating formation in a colloidal suspension of silver nanoparticles. Opt. Lett. 31, 447-449 (2006).

66. Liu, G. L., Kim, J., Lu, Y. \& Lee, L. P. Optofluidic control using photothermal nanoparticles. Nature Mater. 5, 27-32 (2006).

67. Whitesides, G. et al. Dynamic control of liquid-core/liquid-cladding optical waveguides Proc. Natl Acad. Sci. USA 34, 12434-12438 (2004).

68. Erickson, D. Spectrographic microfluidic memory. Proc. ICMM June 13-15 2005, Canada.

Acknowledgements This work is funded by the Defense Advanced Research Projects Agency (DARPA) Center for Optofluidic Integration, USA. We thank J. Adleman, X Heng, Y. Fainman and D. Erickson for numerous discussions and their assistance.

Author Information Reprints and permissions information is available at npg.nature.com/reprintsandpermissions. The authors declare competing financial interests: details accompany the paper at www.nature.com/nature. Correspondence should be addressed to D.P. (psaltis@sunoptics.caltech.edu). 